\title{
Sound Navigation Aid System for the Vision Impaired
}

\author{
M. S. Muhammad, Y. W. Thoo, and S. M. W. Masra \\ Department of Electronic Engineering, \\ Universiti Malaysia Sarawak (UNIMAS), \\ 94300 Kota Samarahan, Sarawak, Malaysia.
}

\begin{abstract}
Visually impaired or blind people are someone who lost their ability to see. They usually depend on guide cane or guide dog to assist them navigates their environment. However, guide cane requires the blind to scan the surrounding manually with their hand while guide dog is expensive and has a short life span. There are many researches on creating better navigation aid for blind people. Instead of using vibration or alarm to notify the distance of the obstacles, this research proposed the application of sound notification for the blind. This system is developed using MATLAB r2010a with the integrated webcam of the laptop and a laser pointer. The laser pointer acts as a reference point so that object's distance can be calculated from the captured image by the webcam. This system manages to calculate distance for any color of object under any level of brightness condition during day and night time. It also possesses human head movement ability to turn left and right, up and down. This system managed to detect the obstacles up to a distance of 432 centimeters, which is sufficient to prevent the blind from bumping into the obstacles. The calculated distance is then converted to sound in order to notify the blind how far the object is away from them.
\end{abstract}

Keywords—blind; navigation aid; range finder; sound imaging

\section{INTRODUCTION}

According to World Health Organization (WHO) statistics, there are 285 million visually impaired people worldwide with 39 million are blind and 246 million have low vision [1]. Blindness is defined to be incapability to see or lacking of the sense of sight [2]. The leading causes of chronic blindness include cataract, glaucoma, age-related macular degeneration, corneal opacities, diabetic retinopathy, trachoma and eyes' conditions in children which caused by vitamin A deficiency. Blindness caused by infection is decreasing due to the result of public health action. However, age-related blindness is increasing throughout the world due to uncontrolled diabetes [2].

According to International Classification of Diseases - 10 (Update and Revision 2006), visual function comprises of four levels which are normal vision, moderate visual impairment, severe visual impairment and blindness [1]. The global foremost causes of visual impairment are due to uncorrected refractive errors $(43 \%)$, cataract $(33 \%)$ and glaucoma $(2 \%)$. Nonetheless, $80 \%$ of all visual impairment can be prevented or cured [3].
In the last 20 years, government worldwide has launched national programs to prevent and control visual impairment. Eye care services are also gradually incorporated into primary and secondary health care system. Campaigns to invoke consciousness including school-based education and stronger international partnerships with engagement of the private sector and civil society are implemented too.

Although blind people can read printed matter in braille, their most apparent problem faced is to navigate around without bumping into unexpected obstacles [4]. A mistake in displacement orientation may put a blind person in jeopardy for an accident which might involve other persons [5].

Due to blind people's enhanced hearing system, the idea of employing moving images distance conversion into real time sounds is thus brought up. With the aid of a webcam, blind people's sense of sight is brought back in a better manner. A program is developed using MATLAB software to identify objects and obstacles such as vehicles, furniture, people, tree and so on. The developed program calculates the distance of the obstacles from the user, and is then transformed to sound that will assist the blind people to move around without the use of a guide cane or any help.

\section{RELATED WORKS}

Blind people usually utilizes white cane and guide dog as their navigation aids to travel around their environment $[6,7$, 8], but they preferred better navigation aid which is simple, convenience to be carried around, light weight and most importantly cheap and deliver greater obstacles information to them. Thus, Electronics Travel Aids (ETAs) is introduced [9, 10, 11]. Navbelt, vOICe, NAVI and VAS are among popular navigation aids which employ image to sound conversion.

Navbelt is a device which applies mobile robotic technology to assist blind people [12, 13]. It comprises of a belt with eight ultrasonic sensors mounted in front the user and a portable computer which are worn as backpack [14]. These sensors provide a total scan of $120^{\circ}$ range. The obstacle's direction is directed by the perceived spatial direction of the signal while its distance is shown by the signal's volume. It is not designed for obstacle detection and avoidance, climb up or down stairs and too heavy to be carried around when necessary. This system's drawbacks are the bulky prototype and user are required go through extensive training. 